\section{Prevalência de atividade física no lazer e fatores associados: estudo de base populacional em São Paulo, Brasil, 2008-2009}

\author{
Prevalence of leisure-time physical activity and \\ associated factors: a population-based study \\ in São Paulo, Brazil, 2008-2009
}

Clóvis Arlindo de Sousa 1

Chester Luiz Galvão César 2 Marilisa Berti de Azevedo Barros ${ }^{3}$ Luana Carandina 4 Moisés Goldbaum 5 Dirce Maria Lobo Marchioni 2 Regina Mara Fisberg 2

\footnotetext{
${ }_{1}^{1}$ Central de Cursos de Pósgraduação, Universidade Gama Filho, São Paulo, Brasil.

2 Faculdade de Saúde Pública, Universidade de São Paulo, São Paulo, Brasil. 3 Faculdade de Ciências Médicas, Universidade Estadual de Campinas, Campinas, Brasil. 4 Faculdade de Medicina de Botucatu, Universidade Estadual Paulista Júlio de Mesquita Filho, Botucatu, Brasil.

5 Faculdade de Medicina, Universidade de São Paulo, São Paulo, Brasil.

Correspondência C. A. Sousa Central de Cursos de Pósgraduação, Universidade Gama Filho.

Rua Treze de Maio 681, São Paulo, SP 01327-000, Brasil.

clovissousa@usp.br
}

\begin{abstract}
The purpose of this study was to ascertain the prevalence of self-reported leisure-time physical activity and related factors in the city of São Paulo, Brazil, 2008-2009. A population- based crosssectional study interviewed 2,691 individuals of both sexes, 12 years or older. A two-stage cluster (census tract, household) random sample provided data using home interviews in 2008 and 2009. Leisure-time physical activity was measured with IPAQ, long version. Complex sample-adjusted descriptive statistics provided prevalence estimates, chi-square tests screened associations, and prevalence ratios (PR) expressed effects. Multiple Poisson regression was used to ascertain adjusted effects, and design effects were calculated. Of the interviewees, $16.4 \%$ (95\%CI: 14.3-18.7) reported leisure-time physical activity. The findings indicate the importance of encouraging leisure-time physical activity, which was associated with male sex, higher income, younger age (12 to 29 years), not smoking, and not reporting frequent fatigue.
\end{abstract}

Motor Activity; Leisure Activities; Life Style

\section{Resumo}

O objetivo deste estudo foi estimar a prevalência e os fatores associados à atividade física no lazer. Estudo transversal, de base populacional, utilizando-se os dados do Inquérito de Saúde no Município de São Paulo 2008 (ISA-Capital 2008), com 2.691 indivíduos de ambos os sexos e com 12 anos ou mais. As informações foram coletadas por entrevistas domiciliares e os participantes foram selecionados com base em amostragem probabilística por conglomerados em dois estágios. Para estimar atividade física no lazer foi utilizado o IPAQ, versão longa. Foram usados estatísticas descritivas, testes de associação pelo qui-quadrado, razões de prevalência e intervalos de 95\% de confiança. Para análise ajustada foi realizada regressão múltipla de Poisson. Dos entrevistados, 16,4\% referiram praticar atividade física suficiente no lazer. Os achados deste trabalho apontam a importância de incentivar a atividade física no lazer, associada ao sexo masculino, maior renda, idades mais jovens entre 12 e 29 anos, não uso do cigarro e referir não sentirse cansado(a) frequentemente.

Atividade Motora; Atividades Recreativas; Estilo de Vida 


\section{Introdução}

Estimativas globais indicam que a inatividade física é responsável por $6 \%$ das ocorrências de doenças cardiovasculares, $7 \%$ das ocorrências de diabetes do tipo 2, 10\% dos casos de câncer de mama e de cólon, e por $9 \%$ das mortes prematuras, ou seja, mais de 5,3 milhões das 57 milhões de mortes ocorridas no mundo em 2008 1,2. Nesse sentido, a atividade física regular é considerada determinante para a redução do risco de doenças crônicas e de mortalidade por todas as causas 3,4 . Outros benefícios sugerem a redução do estresse e dos sintomas da depressão e o aumento da sensação de bem-estar, envolvendo maiores níveis de autoconfiança e satisfação pessoal 5,6.

O sistema de Vigilância de Fatores de Risco e Proteção para Doenças Crônicas por Inquérito Telefônico (VIGITEL) de 2010, com amostra de mais de 54 mil indivíduos com 18 anos ou mais, estimou a prevalência de atividade física das capitais de todos os estados brasileiros e no Distrito Federal em 14,9\% (IC95\%: 14,1-15,8), maior em homens $(18,6 \%)$ do que em mulheres $(11,7 \%)$. Foi considerada atividade física suficiente no lazer a prática de pelo menos 30 minutos diários de atividade física de intensidade leve ou moderada em cinco ou mais dias da semana, ou a prática de pelo menos 20 minutos diários de atividade física de intensidade vigorosa em três ou mais dias da semana. A prevalência de ativos no lazer aumentou com a escolaridade e diminuiu com a idade 7 .

A atividade física, por ser um comportamento humano, é considerada complexa de ser entendida e de ser medida com precisão. Como determinantes desse complexo comportamento, duas categorias podem influenciar os níveis de atividade física: as características individuais (motivações, autoeficácia, habilidades motoras e outros comportamentos de saúde) e as características ambientais (acesso ao trabalho ou espaços de lazer, custos, barreiras de disponibilidade de tempo e suporte sociocultural). São quatro os contextos ou domínios em que as pessoas podem ser fisicamente ativas: no trabalho, deslocamento diário, atividades domésticas e no lazer. Uma pessoa pode ser considerada ativa ou inativa, em um ou mais desses domínios, quando atende ou não a uma recomendação. Nesse sentido, considera-se como inativa em termos globais a pessoa classificada como inativa nos quatro domínios de atividade física 8,9 .

De acordo com estudos conduzidos no Brasil, os fatores mais fortemente associados à inatividade física no lazer foram: sexo feminino, menor renda familiar, menor escolaridade, maior faixa etária, inexistência de local para praticar ativi- dades físicas próximo à residência e presença de obesidade; outros fatores controversos ou associados em alguns estudos foram: presença de tabagismo, incapacidades, doenças crônicas (como diabetes mellitus, doenças cardiovasculares, artrite/reumatismo/artrose, doença respiratória crônica e hipertensão arterial sistêmica), depressão, dificuldades para locomoção, sentirem-se frequentemente cansados, com percepção da saúde ruim e com menor qualidade de vida 10,11, $12,13,14,15,16,17,18,19,20,21,22,23$.

Embora a literatura em epidemiologia da atividade física venha crescendo quantitativamente no Brasil, informações sobre a frequência e a distribuição da prática de atividade física e seus fatores associados ainda são escassas considerando as várias regiões do país e, sobretudo, existem limitações metodológicas que dificultam a comparação entre os estudos. Para o Município de São Paulo existem poucos estudos de base populacional sobre estimação da prevalência e fatores associados para atividade física suficiente no lazer que incluíram adolescentes e idosos 24 .

Considerando a relevância desse problema no país, o objetivo deste trabalho foi estimar a prevalência e os fatores associados à atividade física no lazer em pessoas com 12 anos ou mais no Município de São Paulo.

\section{Métodos}

Estudo transversal, de base populacional, utilizando-se os dados do Inquérito de Saúde no Município de São Paulo 2008 (ISA-Capital 2008), realizado de outubro 2008 a maio de 2009. A população do estudo é a residente em domicílios particulares do Município de São Paulo e setores de situação urbana: área urbanizada, não urbanizada e urbanizada isolada, e dos tipos: comum e especial subnormal (favela), segundo classificação do Instituto Brasileiro de Geografia e Estatística (IBGE). Com base nos objetivos do projeto ISA-Capital 2008, foram fixados oito domínios de estudo formados pelos grupos sexo/idade: menores de um ano, de 1 a 11 anos, mulheres de 12 a 19, 20 a 59 e 60 ou mais, e homens, nas mesmas faixas etárias. A amostra total foi de 3.271 pessoas, e para este estudo foram selecionados os indivíduos com 12 anos ou mais, de ambos os sexos, perfazendo um total de 2.691 .

Os participantes foram selecionados por meio de amostragem probabilística por conglomerados em dois estágios: setores censitários e domicílios. Os números de domicílios visitados foram 90, 13, 30 e 45 para, respectivamente, os grupos de menores de um ano, de crianças 
e adultos, de adolescentes e de idosos, levando a diferentes probabilidades de seleção. Para atingir o número de domicílios foram sorteados 70 setores com base na Pesquisa Nacional por Amostra de Domicílios (PNAD-2002), que registrou 267 setores censitários urbanos no município. Detalhes da amostragem podem ser obtidos em Alves \& Escuder 25. As informações foram obtidas por meio de questionário estruturado em 21 blocos temáticos com a maioria das questões fechadas, aplicado à pessoa sorteada 26 . As entrevistas foram realizadas por pessoal treinado e supervisionado durante toda a duração do inquérito. Entrevistas por telefone ou diretamente no domicílio foram realizadas em amostra aleatória de $5 \%$ das entrevistas para assegurar o controle de qualidade na coleta de dados. A taxa de não resposta foi de 22,5\%, e 7,3\% de domicílios vagos ou cujos moradores se recusaram a informar se havia alguém da faixa etária de interesse residindo ali.

A variável dependente utilizada foi atividade física suficiente no lazer (ativo/inativo), definida como a prática de atividade física por pelo menos 30 minutos diários em cinco dias na semana em intensidade moderada, ou pelo menos $20 \mathrm{mi}$ nutos diários em três dias na semana em intensidade vigorosa. Foram utilizadas as perguntas referentes ao período de lazer do Questionário Internacional de Atividades Físicas (International Physical Activity Questionnaire - IPAQ), versão longa 27.

As variáveis independentes selecionadas para análise foram: variáveis sociodemográficas e econômicas: sexo, idade, cor da pele, situação conjugal, escolaridade do chefe da família, renda do chefe da família, caracterização do domicílio, tipo de moradia e número de cômodos; variáveis de estilo de vida: tabagismo, número de cigarros por dia, tempo de fumo e dependência alcoólica avaliada por meio do teste Cut Down, Annoyed by Criticism, Guilty and Eyeopener (CAGE); e variáveis de condições de saúde: índice de massa corporal (IMC) calculado com dados de peso e estatura referidos, instrumento Self-Report Questionnaire-SRQ-20 (dorme mal, dificuldade para as atividades diárias, cansado(a) o tempo todo e cansa com facilidade), problemas de saúde nos 15 dias anteriores à entrevista, e relatos da presença de asma, alergia, rinite alérgica, sinusite, anemia, doença de coluna/costas, osteoporose, artrite/reumatismo/artrose, doença renal crônica, depressão/ansiedade/problemas emocionais, doença pulmonar obstrutiva crônica, diabetes, hipertensão arterial sistêmica, acidente vascular cerebral e doença do coração.

A associação entre as variáveis independentes e atividade física no lazer foi verificada na análise bivariada pelo teste qui-quadrado com nível de significância de $5 \%$. Foram utilizadas razões de prevalência e intervalos de $95 \%$ de confiança, e para análise ajustada foi realizada regressão múltipla de Poisson, utilizando o método stepwise forward selection para a modelagem estatística. O modelo foi iniciado pela variável mais significativa, do ponto de vista estatístico, e, a seguir, foram acrescentadas as outras, uma a uma. A variável permanecia no modelo sempre que fosse estatisticamente significativa e/ou ajustasse melhor o modelo. No modelo múltiplo foram consideradas as variáveis que tiveram um $\mathrm{p}<0,20$ na análise bivariada e permaneceram no modelo apenas aquelas com $\mathrm{p}<0,05$. Interações entre as variáveis do modelo final foram examinadas. Em todas as análises considerou-se o efeito do desenho amostral para análise de inquéritos baseados em delineamentos complexos. Utilizou-se o programa SPSS 16.0 (SPSS Corp., Chicago, Estados Unidos), que permite incorporar os pesos distintos das observações.

Os participantes assinaram um Termo de Consentimento Livre e Esclarecido no qual eram explicados os objetivos da pesquisa e as informações que seriam solicitadas, sendo garantida a confidencialidade das informações obtidas. $\mathrm{O}$ protocolo de pesquisa foi aprovado pelo Comitê de Ética em Pesquisa da Faculdade de Saúde Pública da Universidade de São Paulo (processo no $381 / 2010$ ).

\section{Resultados}

A prevalência estimada para atividade física suficiente no lazer em pessoas com 12 anos ou mais foi de 16,4\% (IC95\%: 14,3-18,7\%), entre 12 e 19 foi de $25 \%$ (IC95\%: 20,7-29,7\%), e entre 20 anos e mais foi de 14,8\% (IC95\%: 12,6-17,4\%). Dos 2.691 entrevistados com 12 anos ou mais, $1.524(53,1 \%)$ eram do sexo feminino e 1.626 (62,5\%) de cor da pele branca. Quanto aos chefes de família, 40\% tinham escolaridade fundamental, 32,9\% deles possuíam Ensino Médio e 71,7\% tinham renda de até 3 salários mínimos. Moravam em domicílios caracterizados como casa 88,2\%, 64,3\% em moradia própria e $66,3 \%$ residiam em domicílios com 4 cômodos ou mais (Tabela 1).

Para análise bruta, segundo as variáveis sociodemográficas e econômicas, a prevalência de atividade física no lazer foi de $20,3 \%$ para o sexo masculino e de $12,9 \%$ para o feminino. A faixa etária entre 12 e 19 anos apresentou maior prevalência de atividade física (25\%) em comparação com as idades entre 40 e 49 anos $(12,1 \%)$. A prática de atividade física foi maior para os que têm a cor da pele branca $(17,8 \%)$ em 
Distribuição da amostra e prevalência de atividade física no lazer em pessoas com 12 anos ou mais, segundo variáveis sociodemográficas e econômicas. São Paulo, Brasil, 2008-2009.

\begin{tabular}{|c|c|c|c|c|c|}
\hline Variáveis e categorias & $\mathrm{n}$ & $\%$ & Prevalência (\%) & Valor de $p$ * & RP (IC95\%) \\
\hline Sexo & & & & $<0,001$ & \\
\hline Feminino & 1.524 & 53,1 & 12,9 & & 1,00 \\
\hline Masculino & 1.167 & 46,9 & 20,3 & & $1,71(1,30-2,26)$ \\
\hline Idade (anos) & & & & 0,002 & \\
\hline $12-19$ & 605 & 15,1 & 25,0 & & $2,42(1,54-3,79)$ \\
\hline $20-29$ & 348 & 21,8 & 18,5 & & $1,64(1,12-2,52)$ \\
\hline $30-39$ & 297 & 18,5 & 14,6 & & $1,24(0,76-2,03)$ \\
\hline $40-49$ & 295 & 17,6 & 12,1 & & 1,00 \\
\hline $50-59$ & 222 & 13,2 & 17,0 & & $1,48(0,90-2,44)$ \\
\hline $60-69$ & 443 & 6,8 & 12,5 & & $1,04(0,62-1,75)$ \\
\hline 70 e mais & 481 & 7,1 & 12,9 & & $1,08(0,63-1,85)$ \\
\hline Cor da pele & & & & 0,030 & \\
\hline Preta/Parda & 1.010 & 37,5 & 13,6 & & 1,00 \\
\hline Branca & 1.626 & 62,5 & 17,8 & & $1,37(1,03-1,82)$ \\
\hline Situação conjugal & & & & 0,115 & \\
\hline Sem cônjuge & 888 & 29,1 & 13,8 & & 1,00 \\
\hline Com cônjuge & 1.766 & 70,9 & 17,3 & & $1,31(0,93-1,84)$ \\
\hline Escolaridade do chefe da família & & & & $<0,001$ & \\
\hline Nenhuma & 157 & 3,5 & 9,0 & & 1,00 \\
\hline Fundamental & 1.443 & 40,6 & 11,1 & & $1,27(0,56-2,87)$ \\
\hline Médio & 662 & 32,9 & 17,0 & & $2,07(0,85-5,05)$ \\
\hline Superior & 391 & 23,0 & 25,5 & & $3,48(1,37-8,85)$ \\
\hline Renda do chefe da família (salários mínimos) ** & & & & $<0,001$ & \\
\hline$\leq 1$ & 773 & 26,2 & 10,6 & & 1,00 \\
\hline $1-3$ & 1.115 & 45,5 & 13,0 & & $1,25(0,89-1,76)$ \\
\hline $3-6$ & 331 & 17,9 & 16,5 & & $1,66(1,08-2,56)$ \\
\hline$>6$ & 162 & 10,4 & 30,9 & & $3,76(2,37-5,95)$ \\
\hline Caracterização do domicílio & & & & $<0,001$ & \\
\hline Casa & 2.417 & 88,2 & 14,8 & & 1,00 \\
\hline Apartamento & 252 & 11,8 & 29,2 & & $2,38(1,62-3,50)$ \\
\hline Moradia & & & & 0,318 & \\
\hline Não própria & 856 & 35,7 & 15,2 & & 1,00 \\
\hline Própria & 1.816 & 64,3 & 17,0 & & $1,14(0,88-1,49)$ \\
\hline Número de cômodos no domicílio & & & & 0,260 & \\
\hline $1-3$ & 880 & 33,7 & 14,4 & & 1,00 \\
\hline 4 e mais & 1.806 & 66,3 & 17,4 & & $1,25(0,85-1,84)$ \\
\hline
\end{tabular}

IC95\%: intervalo de 95\% de confiança; RP: razão de prevalência.

* Teste de qui-quadrado;

** 1 salário mínimo $=\mathrm{R} \$ 622,00$.

comparação aos de pele preta/parda (13,6\%), e sua prevalência também foi maior à medida que a escolaridade e a renda do chefe da família aumentaram. Houve maior prevalência de atividade física entre os indivíduos que residiam em apartamentos $(29,2 \%)$ quando comparados com os que viviam em casas $(14,8 \%)$. A situação conjugal, o número de cômodos no domicílio e o tipo de moradia, não apresentaram associação significativa com a prática de atividade física de lazer (Tabela 1).

Segundo as variáveis de estilo de vida, verificou-se a prevalência de tabagismo de 19,3\%, sendo que a prevalência de atividade física nos fu- 
mantes foi de $9,8 \%$, quando comparada a $18,3 \%$ entre os que nunca fumaram. A prevalência de atividade física suficiente no lazer apresentou efeito dose/resposta à proporção que diminui o número de cigarros fumados por dia, e a prevalência de dependência alcoólica foi de 9,9\%, não apresentando associação com atividade física (Tabela 2).

Entre as variáveis de condições de saúde, a prevalência de obesidade foi de $12,8 \%$ e de sobrepeso e obesidade $45 \%$. Não houve associação entre prática de atividade física de lazer e IMC. Houve associação entre prevalência de atividade física e referir não possuir dificuldades para as atividades diárias, não sentir-se cansado(a) o tempo todo, cansar-se com facilidade, e problemas de saúde nos últimos 15 dias anteriores à entrevista. Ainda na análise bruta, houve associação entre atividade física e anemia, artri- te/reumatismo/artrose e depressão/ansiedade/ problemas emocionais (Tabela 2).

As variáveis que permaneceram associadas foram: idade $(p=0,05)$, sexo $(p=0,024)$, renda do chefe da família $(\mathrm{p}<0,001)$, número de cigarros fumados por dia ( $\mathrm{p}=0,043$ ) e cansado(a) o tempo todo $(\mathrm{p}<0,001)$ (Tabela 3$)$. Na estratificação para idades de 12 a 19 anos, permaneceram no modelo final somente sexo $(\mathrm{p}<0,001)$ e renda do chefe da família ( $p<0,001$ ), com aumento da força de associação para sexo, ou seja, existe mais diferença (entre sexos) de prática de atividade física entre os adolescentes do que entre os adultos com 20 anos mais. Ou pode-se interpretar que as adolescentes, quando se comparam com os adolescentes, são mais inativas fisicamente em relação às mulheres com 20 anos e mais com seus pares masculinos. Para a faixa etária de 20 anos e mais, as variáveis independentemente

Tabela 2

Distribuição da amostra e prevalência de atividade física no lazer em pessoas com 12 anos ou mais, segundo variáveis de estilo de vida e condições de saúde. São Paulo, Brasil, 2008-2009.

\begin{tabular}{|c|c|c|c|c|c|}
\hline Variáveis e categorias & $\mathbf{n}$ & $\%$ & Prevalência (\%) & Valor de $p$ * & RP (IC95\%) \\
\hline Tabagismo & & & & 0,004 & \\
\hline Fumante & 422 & 19,3 & 9,8 & & 1,00 \\
\hline Ex-fumante & 479 & 16,3 & 16,6 & & $1,84(1,05-3,23)$ \\
\hline Nunca fumou & 1.789 & 64,4 & 18,3 & & $2,06(1,29-3,30)$ \\
\hline Número de cigarros por dia & & & & 0,002 & \\
\hline$>20$ & 50 & 2,2 & 8,5 & & 1,00 \\
\hline$\leq 20$ & 363 & 16,8 & 17,9 & & $2,36(1,43-3,86)$ \\
\hline Nenhum & 2.278 & 81,0 & 20,9 & & $2,86(1,13-7,25)$ \\
\hline Tempo de fumo (anos) ** & & & & 0,831 & \\
\hline$>30$ & 536 & 44,1 & 12,3 & & 1,00 \\
\hline$\leq 30$ & 360 & 55,9 & 13,4 & & $1,12(0,39-3,22)$ \\
\hline Dependência de bebida alcoólica (CAGE) & & & & 0,212 & \\
\hline Dependente & 123 & 9,9 & 12,9 & & 1,00 \\
\hline Não dependente & 1.146 & 90,1 & 17,4 & & $1,42(0,82-2,48)$ \\
\hline Índice de massa corporal $\left(\mathrm{kg} / \mathrm{m}^{2}\right)$ & & & & 0,513 & \\
\hline Obeso & 346 & 12,8 & 14,0 & & 1,00 \\
\hline Sobrepeso & 807 & 32,2 & 15,8 & & $1,15(0,74-1,80)$ \\
\hline Peso normal & 1.229 & 49,6 & 17,7 & & $1,32(0,87-2,00)$ \\
\hline Abaixo do peso & 175 & 5,4 & 17,1 & & $1,26(0,71-2,26)$ \\
\hline Dorme mal & & & & 0,037 & \\
\hline $\operatorname{Sim}$ & 783 & 32,2 & 12,0 & & 1,00 \\
\hline Não & 1.510 & 67,8 & 16,9 & & $1,49(1,02-2,17)$ \\
\hline Dificuldade para as atividades diárias ** & & & & 0,001 & \\
\hline Sim & 377 & 14,9 & 8,0 & & 1,00 \\
\hline Não & 1.916 & 85,1 & 16,6 & & $2,28(1,43-3,64)$ \\
\hline
\end{tabular}




\begin{tabular}{|c|c|c|c|c|c|}
\hline Variáveis e categorias & n & $\%$ & Prevalência (\%) & Valor de $p$ * & RP (IC95\%) \\
\hline Cansado(a) o tempo todo ** & & & & $<0,001$ & \\
\hline $\operatorname{Sim}$ & 440 & 17,8 & 5,3 & & 1,00 \\
\hline Não & 1.853 & 82,2 & 17,5 & & $3,73(2,18-6,37)$ \\
\hline Cansa com facilidade ** & & & & $<0,001$ & \\
\hline $\operatorname{Sim}$ & 601 & 22,6 & 7,0 & & 1,00 \\
\hline Não & 1.692 & 77,4 & 17,7 & & $2,81(1,91-4,14)$ \\
\hline Problemas de saúde nos últimos 15 dias & & & & 0,034 & \\
\hline Sim & 571 & 19,7 & 12,6 & & 1,00 \\
\hline Não & 2.120 & 80,3 & 17,3 & & $1,45(1,03-2,04)$ \\
\hline \multicolumn{6}{|l|}{ Relato das seguintes doenças/condições } \\
\hline Asma & 138 & 7,2 & 17,3 & 0,848 & $1,06(0,54-2,10)$ \\
\hline Alergia & 579 & 17,0 & 14,1 & 0,289 & $0,80(0,54-1,20)$ \\
\hline Rinite & 513 & 22,0 & 6,9 & 0,228 & $1,20(0,88-1,64)$ \\
\hline Sinusite & 392 & 16,7 & 16,7 & 0,906 & $1,03(0,68-1,57)$ \\
\hline Anemia & 98 & 3,4 & 6,0 & 0,044 & $0,31(0,10-0,97)$ \\
\hline Doença de coluna/costas & 438 & 12,6 & 15,9 & 0,872 & $0,96(0,60-1,54)$ \\
\hline Osteoporose ** & 161 & 2,8 & 14,2 & 0,884 & $1,05(0,48-2,31)$ \\
\hline Artrite/Reumatismo/Artrose ** & 329 & 7,5 & 8,7 & 0,016 & $0,52(0,31-0,89)$ \\
\hline Doença renal crônica & 77 & 2,5 & 6,4 & 0,098 & $0,34(0,10-1,23)$ \\
\hline Depressão/Ansiedade/Problemas emocionais ** & 538 & 18,2 & 11,5 & 0,020 & $0,66(0,47-0,94)$ \\
\hline Doença pulmonar obstrutiva crônica ** & 121 & 3,9 & 11,0 & 0,205 & $0,64(0,32-1,28)$ \\
\hline Diabetes & 246 & 5,5 & 15,0 & 0,675 & $0,90(0,54-1,49)$ \\
\hline Hipertensão arterial sistêmica ** & 711 & 18,3 & 11,4 & 0,067 & $0,68(0,44-1,03)$ \\
\hline Acidente vascular cerebral & 69 & 1,5 & 21,6 & 0,431 & $1,41(0,59-3,38)$ \\
\hline Doença do coração & 201 & 4,5 & 11,2 & 0,142 & $0,63(0,34-1,17)$ \\
\hline
\end{tabular}

CAGE: Cut Down, Annoyed by Criticism, Guilty and Eyeopener; IC95\%: intervalo de 95\% de confiança; RP: razão de prevalência.

* Teste de qui-quadrado;

** Ajustado por idade.

associadas à pratica de atividade física de lazer foram: sexo ( $p=0,047)$, renda do chefe da família $(\mathrm{p}<0,001)$, número de cigarros fumados por dia ( $p=0,036)$ e cansado(a) o tempo todo ( $p<0,001)$. Comparada a análise geral (12 anos e mais), a idade perdeu associação e elevou a força de associação para renda do chefe da família, número de cigarros por dia e cansaço frequente (Tabela 4). Não houve interação entre as variáveis dos modelos finais.

\section{Discussão}

As recomendações atuais da saúde pública para a prática de atividade física suficiente, segundo o American College of Sports Medicine (ACSM) e a American Heart Association (AHA), envolvem pelo menos 30 minutos diários de atividade física em intensidade moderada em cinco ou mais dias da semana, ou a prática de pelo menos 20 minutos diários de atividade física em intensidade vigorosa em três ou mais dias na semana. Os exercícios de força e resistência muscular e de flexibilidade e equilíbrio também são importantes, pois melhoram essas características motoras, o que para os idosos podem reduzir o risco de quedas por aumentar as capacidades físicas 4,28.

A prática de atividade física no lazer é somente uma das dimensões da atividade física. As recomendações de saúde pública enfatizam a importância da atividade física acumulada em vários cenários da vida, além do lazer, nas atividades ocupacionais, domésticas e no deslocamento diário ${ }^{4}$. Contudo, a medida da atividade física no lazer vem ganhando importância por seu caráter opcional e prazeroso e, sobretudo por apresentar associações mais consistentes com os fatores de risco de doenças cardiovasculares quando comparada às atividades ocupacionais 29 .

Segundo duas recentes revisões sistemáticas sobre atividade física no Brasil, foram realizados 
Modelo de regressão múltipla de Poisson para atividade física no lazer em pessoas com 12 anos ou mais. São Paulo, Brasil 2008-2009.

\begin{tabular}{|c|c|c|c|c|}
\hline Variáveis & RP (IC95\%) & Erro-padrão & Valor de p & Efeito do desenho \\
\hline \multicolumn{5}{|l|}{ Idade (anos) } \\
\hline $12-19$ & $1,90(1,16-3,50)$ & 0,31 & 0,050 & 1,11 \\
\hline $20-29$ & $1,71(1,04-3,13)$ & 0,28 & & 1,84 \\
\hline $30-39$ & $1,45(0,88-2,39)$ & 0,29 & & 1,21 \\
\hline $40-49$ & 1,00 & & & \\
\hline $50-59$ & $1,66(0,93-2,95)$ & 0,31 & & 1,50 \\
\hline $60-69$ & $1,12(0,64-1,94)$ & 0,36 & & 0,79 \\
\hline 70 e mais & $1,25(0,85-2,29)$ & 0,24 & & 0,93 \\
\hline \multicolumn{5}{|l|}{ Sexo } \\
\hline Feminino & 1,00 & & & \\
\hline Masculino & $1,55(1,06-2,27)$ & 0,19 & 0,024 & 2,04 \\
\hline \multicolumn{5}{|c|}{ Renda do chefe da família (salários mínimos) } \\
\hline$\leq 1$ & 1,00 & & & \\
\hline $1-3$ & $1,42(0,93-2,18)$ & 0,29 & $<0,001$ & 1,28 \\
\hline $3-6$ & $1,86(1,10-3,13)$ & 0,30 & & 1,40 \\
\hline$>6$ & $4,13(2,31-7,35)$ & 0,30 & & 1,70 \\
\hline \multicolumn{5}{|c|}{ Número de cigarros por dia } \\
\hline$>20$ & 1,00 & & & \\
\hline$\leq 20$ & $1,51(0,84-2,71)$ & 0,36 & 0,043 & 1,95 \\
\hline Nenhum & $3,09(1,06-9,09)$ & 0,41 & & 1,97 \\
\hline \multicolumn{5}{|c|}{ Cansado(a) o tempo todo } \\
\hline $\operatorname{Sim}$ & 1,00 & & & \\
\hline Não & $3,32(1,89-5,80)$ & 0,32 & $<0,001$ & 1,06 \\
\hline
\end{tabular}

IC95\%: intervalo de 95\% de confiança; RP: razão de prevalência.

68 estudos acerca do tema em amostras aleatórias, sendo que apenas nove foram publicados antes do ano 2000. Houve uma tendência de aumento de publicações nos últimos 10 anos, com grande concentração de trabalhos nas regiões Sul e Sudeste, e na maioria dos estudos a atividade física na população foi mensurada por meio de questionários, sendo o domínio mais estudado o lazer 24,30.

De acordo com Hallal et al. ${ }^{30}$, em estudo de revisão sistemática sobre atividade física no Brasil, identificou-se a utilização de 26 diferentes definições operacionais da variável sedentarismo. Em virtude dessa variabilidade de critérios, muitos trabalhos apontaram uma prevalência de sedentarismo no Brasil entre 26,7\% e 78,2\%.

Com relação à prevalência da atividade física de lazer no Brasil, outro importante estudo de âmbito nacional identificou níveis semelhantes ao presente trabalho, mostrando que para a cidade de São Paulo em 2010, 13,7\% (IC95\%: 11,2$16,1 \%$ ) dos adultos eram ativos no lazer. A faixa etária de 18 a 24 anos apresentou maior prevalência $(20,4 \%)$ de atividade física quando comparada às idades de 65 e mais (12,2\%). Quanto à distribuição por cidade, a menor prevalência de atividade física no lazer foi observada em Rio Branco, Acre (11,3\%), e a maior em Brasília, Distrito Federal $(22,4 \%) 7$. Outro estudo realizado em São Paulo verificou a prevalência de 22,5\% de atividade física suficiente no lazer em pessoas de 18 a 65 anos de idade 31 .

Estudos conduzidos no Brasil com a população adulta demonstraram que a prevalência de atividade física no lazer é maior em homens do que em mulheres, entre os mais jovens, nos grupos de maior renda e de nível educacional mais elevado, similar ao encontrado no presente trabalho 1,2,10,16,32. O sexo masculino apresentou razão de prevalência 1,55 vez maior para atividade física no lazer em comparação ao feminino. Para as idades entre 12 e 19 anos, o sexo masculino obteve razão de prevalência 2,5 vezes maior em comparação ao feminino, e para a faixa etária 
Modelo de regressão múltipla de Poisson para atividade física no lazer em pessoas entre 12 e 19 anos e com 20 anos e mais. São Paulo, Brasil, 2008-2009.

\begin{tabular}{|c|c|c|c|c|}
\hline Variáveis & RP (IC95\%) & Erro-padrão & Valor de $\mathrm{p}$ & $\begin{array}{l}\text { Efeito do } \\
\text { desenho }\end{array}$ \\
\hline \multicolumn{5}{|l|}{$12-19$ anos } \\
\hline \multicolumn{5}{|l|}{ Sexo } \\
\hline Feminino & 1,00 & & & \\
\hline Masculino & $2,51(1,65-3,82)$ & 0,21 & $<0,001$ & 1,02 \\
\hline \multicolumn{5}{|c|}{ Renda do chefe da família (salários mínimos) } \\
\hline$\leq 1$ & 1,00 & & & \\
\hline $1-3$ & $1,35(0,83-2,04)$ & 0,49 & $<0,001$ & 1,01 \\
\hline $3-6$ & $1,81(1,05-3,07)$ & 0,48 & & 1,56 \\
\hline$>6$ & $4,05(2,26-7,19)$ & 0,53 & & 1,52 \\
\hline \multicolumn{5}{|l|}{20 anos e mais } \\
\hline \multicolumn{5}{|l|}{ Sexo } \\
\hline Feminino & 1,00 & & & \\
\hline Masculino & $1,51(1,04-2,21)$ & 0,21 & 0,047 & 2,15 \\
\hline \multicolumn{5}{|c|}{ Renda do chefe da família (salários mínimos) } \\
\hline$\leq 1$ & 1,00 & & & \\
\hline $1-3$ & $1,44(0,89-2,31)$ & 0,29 & $<0,001$ & 1,27 \\
\hline $3-6$ & $1,92(1,11-3,32)$ & 0,29 & & 1,31 \\
\hline$>6$ & $4,03(2,26-7,19)$ & 0,30 & & 1,49 \\
\hline \multicolumn{5}{|c|}{ Número de cigarros por dia } \\
\hline$>20$ & 1,00 & & & \\
\hline$\leq 20$ & $1,85(0,95-4,28)$ & 0,52 & 0,036 & 1,97 \\
\hline Nenhum & $3,27(1,41-9,31)$ & 0,56 & & 2,19 \\
\hline \multicolumn{5}{|c|}{ Cansado(a) o tempo todo } \\
\hline Sim & 1,00 & & & \\
\hline Não & $4,02(2,07-7,81)$ & 0,33 & $<0,001$ & 1,13 \\
\hline
\end{tabular}

IC95\%: intervalo de 95\% de confiança; RP: razão de prevalência.

de 20 anos e mais o sexo masculino apresentou razão de prevalência 1,5 vez maior para atividade física de lazer. Fatores culturais e sociais parecem determinar as diferenças com relação à prática de atividade física no lazer de acordo com o sexo. Os homens, em sua maioria, associam os exercícios físicos ao prazer, enquanto as mulheres praticam atividade física por questões de saúde, orientação médica ou por estética. Ain$\mathrm{da}$, os homens se envolvem mais em atividades em grupos, como a prática de esportes coletivos, enquanto as mulheres preferem atividades individuais como caminhar, correr e andar de bicicleta. Outra explicação pode ser o fato de muitas mulheres ainda serem responsáveis pelo trabalho doméstico, o que pode diminuir o nível de atividade física no contexto de lazer 18,31,33,34. Os achados de que os homens praticam mais atividade física no lazer do que as mulheres mostram a necessidade de estimular a atividade física de lazer em ambos os sexos, mais especialmente entre as mulheres, sobretudo as adolescentes. A idade também apresentou associação com a prática de atividade física. A faixa etária de 12 a 19 anos apresentou razão de prevalência 1,9 vez maior para atividade física no lazer em comparação com a faixa etária entre 40 e 49 anos, e a de 20 a 29 anos apresentou razão de prevalência 1,7 vez maior em comparação com a de 40 a 49 anos. A maior frequência de atividade física entre homens já foi apontada em outros estudos, assim como entre indivíduos em idades mais jovens $1,2,10,13,16,18,20,31,35$. Outro achado importante constitui o aumento da atividade física no lazer entre pessoas com maior renda familiar, o que pode ser expressão da desigualdade no acesso a espaços físicos e da disponibilidade de tempo livre para a prática de atividades físicas. 
Para o presente estudo, a renda do chefe da família entre três e seis salários mínimos apresentou razão de prevalência 1,9 vez maior para atividade física no lazer se comparada com a renda menor ou igual a um salário mínimo. Para a renda do chefe da família maior que seis salários mínimos a razão de prevalência foi 4 vezes maior em comparação à renda menor ou igual a um salário mínimo. Alguns estudos apresentaram dados similares 10,21,36, demonstrando que o estado de pobreza pode representar um complicador no combate ao sedentarismo. De acordo com Pan et al. 37 , indivíduos com nível educacional mais elevado, assim como com maior renda, possuem mais oportunidades, facilidades e acessos para praticar atividades físicas, além de conviverem num meio social em que a prática é reconhecida por seus pares como favorável à saúde, o que pode facilitar a adesão. Esses grupos tendem a apresentar melhores condições de saúde, melhor suporte social e assimilam com mais facilidade as recomendações e os benefícios da prática regular de atividade física.

Referir não estar cansado(a) o tempo todo apresentou razão de prevalência 3,3 vezes maior para atividade física no lazer quando comparado com aqueles que referiram estar continuamente cansado(a). Na análise estratificada para a faixa entre 12 e 19 anos, essa variável não permaneceu no modelo final e, para 20 anos e mais, referir não estar cansado(a) frequentemente apresentou razão de prevalência 4 vezes maior para atividade física no lazer em relação aos que referiram cansaço frequente. Essa questão refere-se ao conjunto de perguntas do SRQ20 , instrumento de rastreamento para avaliar a ocorrência de transtornos psiquiátricos menores (sofrimento mental), utilizado em outros estudos 10, composto por 20 itens para avaliação nos 30 dias anteriores à entrevista. Este trabalho incluiu também se a pessoa dorme mal, se apresenta dificuldade para as atividades diárias e se cansa com facilidade.

Todas essas questões apresentaram associação estatística significante na análise bruta, e o modelo múltiplo ajustou somente a variável cansado(a) o tempo todo. Além disso, doenças crônicas relacionadas ao coração e ao pulmão podem levar ao cansaço frequente, e em virtude dessa incapacidade e limitação para as atividades diárias provocadas pela doença, os indivíduos podem permanecer em comportamentos sedentários na maior parte do dia para amenizar os sintomas. Essa preferência pelo repouso excessivo é explicada pela sensação de falta de ar e de esgotamento muscular precoce na presença de qualquer intensidade de atividade física 14,38. Florindo et al. 31 verificaram que reportar baixa condição de saúde esteve positivamente associado com o baixo nível de atividade física de lazer, assim como o hábito de fumar. Demonstrando que uma boa percepção de saúde, bons níveis de saúde ou boa condição física e emocional estão associados com maior prática de atividade física em ambientes de lazer.

Não fumar obteve razão de prevalência 3 vezes maior para atividade física no lazer em pessoas com 12 anos e mais se comparado com fumar mais de 20 cigarros por dia, o que perdeu associação na análise estratificada quando se considerou as idades entre 12 e 19 anos. Para 20 anos e mais, não fumar obteve razão de prevalência 3,2 vezes maior para atividade física no lazer se comparado com fumar mais de 20 cigarros por dia. A associação entre consumo de tabaco e inatividade física foi descrita no estudo europeu, em que indivíduos fumadores apresentaram cerca de $50 \%$ mais riscos de serem inativos fisicamente no lazer em relação aos não fumadores 39 . Outros estudos, inclusive conduzidos no Brasil, descreveram associação entre a atividade física insuficiente e o consumo de tabaco, sendo que os fatores de risco para doenças crônicas não transmissíveis, como o tabagismo, bebida alcoólica, dieta inadequada e a obesidade, estão associados à inatividade física no lazer 2,19,31,39,40,41. A associação entre inatividade física e esses comportamentos não saudáveis evidencia a necessidade de intervenções mais amplas dirigidas ao conjunto dos comportamentos e práticas relativas à saúde, sendo que a exposição aos fatores de risco comportamentais, como fumo, má alimentação e sedentarismo, frequentemente se inicia na adolescência e se consolida na vida adulta. Nesse sentido, intervenções de saúde pública devem promover o comportamento saudável desde as idades mais jovens 40,42 .

Com o objetivo de reduzir substancialmente as mortes e doenças em todo o mundo, a Organização Mundial da Saúde (OMS) lançou em maio de 2004, em nível mundial e com o apoio de 192 países, a Estratégia Global em Alimentação Saudável, Atividade Física e Saúde. Um dos principais objetivos é reduzir os fatores de risco para as doenças crônicas não transmissíveis decorrentes da alimentação inadequada e da inatividade física por meio de ações em saúde pública e promoção da saúde e de medidas preventivas. Essa estratégia, que também combina o controle ao tabagismo, representa um reconhecimento mundial oficial da importância desses fatores para a saúde e qualidade de vida da população. Tais acordos internacionais representam um pacto importante para realizar mudanças políticas, econômicas e sociais, nos níveis nacional e internacional, que viabilizem a participação dos profissionais 
de saúde e de outros setores pertinentes, e promovam modos de vida saudáveis 42,43 .

O estudo que analisou a Estratégia Global para Alimentação, Atividade Física e Saúde da OMS, verificou que o documento se refere a um instrumento de promoção geral da saúde para populações e indivíduos, não sendo uma prescrição de tratamento para grupos especiais de risco. Esse fato é importante, pois em um país como o Brasil, onde existem desigualdades sociais e regionais, a promoção da alimentação saudável e da atividade física pressupõe a necessidade de um modelo de atenção à saúde que integre dois lados: a desnutrição e outras doenças associadas à fome, e o sobrepeso/obesidade e as doenças crônicas não transmissíveis associadas 44 . Como mencionado anteriormente, alguns estudos demonstraram que o estado de pobreza pode representar um complicador no combate ao sedentarismo 10,21,36, e essa Estratégia Global não reflete, por exemplo, qual o sentido para um indivíduo que passa fome ou que luta por condições mínimas de reprodução ou de saneamento básico, fazer trinta minutos diários de exercícios aeróbios em cinco ou mais dias na semana. Afinal, por que esse indivíduo gostaria de alcançar uma maior longevidade e/ou se prevenir contra doenças e incapacidades se sua vida não tem nada de qualidade? Essas questões precisam ser consideradas quando se pretende promover saúde e qualidade de vida para a população.

Uma limitação deste estudo diz respeito ao uso de informação referida para prática de atividade física por meio de questionário, o que confere maior margem de erro quando comparado a medidas diretas do nível de atividade física utilizando-se equipamentos de mensuração 30 . Estudos mostram que a informação referida pode superestimar a prevalência de fisicamente ativos em virtude de ser um comportamento socialmente desejável 45. Entretanto, a vantagem da utilização de questionários por autorreferência se refere ao baixo custo e rapidez para obtenção dos dados populacionais quando comparados a outros métodos 30,40 .

Outra limitação é que estudos transversais estão sujeitos ao viés de causalidade reversa, uma vez que exposição e fatores são coletados no mesmo momento, não permitindo identificar possíveis associações causais em virtude do não controle da temporalidade. Para análise dos resultados, esse cuidado é fundamental ao considerar as associações da inatividade física no lazer com o uso do cigarro e com o cansaço frequente.

Inquéritos populacionais de saúde têm um importante papel no conhecimento dos aspectos atuais sobre a situação de morbidade e de saúde da população. As informações em saúde subsidiam ações apoiadas em dados objetivos respaldados por evidência científica. Inquéritos populacionais de saúde periódicos são importantes para gerar informações não obtidas em registros contínuos nacionais, e fundamentais para o planejamento e avaliação das políticas de prevenção e controle de agravos e de promoção da saúde no nível municipal ou regional. Esses inquéritos são importantes para fornecer dados de prevalência sobre (in)atividade física, permitindo o planejamento e implementação de programas de promoção da saúde e incentivo à prática regular de atividade física 46 .

Os achados do presente estudo apontam para a importância de incentivar a prática de atividade física de lazer na população. $\mathrm{O}$ uso do cigarro necessita ser verificado com cautela, mas a associação entre inatividade física e comportamentos não saudáveis, principalmente na vida adulta, demonstra a importância de incentivar o comportamento saudável em todas as idades, sobretudo entre 20 anos e mais. Sentir-se cansado(a) com frequência reforça a importância de estilo de vida ativo para a prevenção de doenças crônicas relacionadas ao coração e ao pulmão, e de problemas de saúde mental de adultos e idosos, que se apresentam associados com inatividade física. Considerando que a prática insuficiente de atividade física é um problema de saúde pública, é necessário que as políticas públicas direcionem e incentivem ações para a prática do lazer ativo, em contrário às atividades passivas e de repouso relacionadas aos comportamentos sedentários e ao uso do cigarro, especialmente para os segmentos menos favorecidos economicamente, do sexo feminino e para os adultos e idosos. 


\section{Resumen}

El objetivo de este estudio fue estimar la prevalencia y los factores asociados a la actividad física durante el ocio. Estudio transversal, de base poblacional, donde se utilizaron los datos de la Encuesta de Salud en el municipio de São Paulo 2008 (ISA-Capital 2008), con 2.691 individuos de ambos sexos y con 12 años o más. La información se recopiló mediante entrevistas domiciliarias y los participantes fueron seleccionados en base al muestreo probabilístico por conglomerados en dos fases. Para estimar la actividad física en el ocio se utilizó el IPAQ, versión larga. Se usaron estadísticas descriptivas, pruebas de asociación por chi-cuadrado, razones de prevalencia e intervalos de un $95 \%$ de confianza. Para el análisis ajustado se realizó una regresión múltiple de Poisson. De los entrevistados, un $16,4 \%$ afirmaron practicar actividad física suficiente en el ocio. Los hallazgos de este trabajo apuntan la importancia de incentivar la actividad física en el ocio, asociada al sexo masculino, mayor renta, edades más jóvenes entre 12 y 29 años, no fumar e informar no sentirse cansado(a) frecuentemente.

Actividad Motora; Actividades Recreativas; Estilo de Vida

\section{Colaboradores}

C. A. Sousa contribuiu com a concepção e redação do artigo e análise e interpretação dos dados. C. L. G. César, M. B. A. Barros, L. Carandina, M. Goldbaum, D. M. L. Marchioni e R. M. Fisberg revisaram o conteúdo intelectual e aprovaram a versão final a ser publicada.

\section{Referências}

1. Kohl 3rd HW, Craig CL, Lambert EV, Inoue S, Alkandari JR, Leetongin G, et al. The pandemic of physical. Lancet 2012; 380:294-305.

2. Hallal PC, Andersen LB, Bull FC, Guthold R, Haskell W, Ekelund U, et al. Global physical activity levels: surveillance progress, pitfalls, and prospects. Lancet 2012; 380:247-57.

3. Blair SN, Kohl III HW, Paffenbarger Junior RS, Clark DG, Kooper KH, Gibbons LW. Physical fitness and all-cause mortality: a prospective study of healthy men and women. JAMA 1989; 262:2395-401.

4. Haskell WL, Lee IM, Pate RR, Powell KE, Blair SN, Franklin BA, et al. Physical activity and public health: updated recommendation for adults from the American College of Sports Medicine and the American Heart Association. Med Sci Sports Exerc 2007; 39:1423-34.

5. Gomes VB, Siqueira KS, Sichieri R. Atividade física em uma amostra probabilística da população do Município do Rio de Janeiro. Cad Saúde Pública 2001; 17:969-76.
6. Pitanga FJ, Lessa I. Prevalência e fatores associados ao sedentarismo no lazer em adultos. Cad Saúde Pública 2005; 21:870-7.

7. Ministério da Saúde. Vigitel Brasil 2010: vigilância de fatores de risco e proteção para doenças crônicas por inquérito telefônico. http://portal.saude. gov.br/portal/saude/profissional/area.cfm?id_ area $=1521$ (acessado em 13/Set/2012).

8. Nahas MV, Garcia LMT. Um pouco de história, desenvolvimentos recentes e perspectivas para a pesquisa em atividade física e saúde no Brasil. Rev Bras Educ Fís Esp 2010; 24:135-48.

9. Sherwood NE, Jeffery RW. The behavioral determinants of exercise: implications for physical activity interventions. Annu Rev Nutr 2000; 20:21-44.

10. Oehlschlaeger MHK, Pinheiro RT, Horta B, Gelatti C, San'Tana P. Prevalence of sedentarism and its associated factors among urban adolescents. Rev Saúde Pública 2004; 38:157-63. 
11. Masson CR, Dias-da-Costa JS, Olinto MTA, Meneghel S, Costa CC, Bairros F, et al. Prevalência de sedentarismo nas mulheres adultas da Cidade de São Leopoldo, Rio Grande do Sul, Brasil. Cad Saúde Pública 2005; 21:1685-95.

12. Baretta E, Baretta M, Peres KG. Nível de atividade física e fatores associados em adultos no Município de Joaçaba, Santa Catarina, Brasil. Cad Saúde Pública 2007; 23:1595-602.

13. Ceschini FL, Romero J, Lima V. Prevalência de inatividade física e fatores associados em bancários. Rev Bras Ciênc Saúde 2007; III:3-11.

14. Benedetti TRB, Borges LJ, Petroski EL, Gonçalves LHT. Atividade física e estado de saúde mental de idosos. Rev Saúde Pública 2008; 42:302-7.

15. Paes MJO, Duarte YAO, Lebrão ML, Santos JLF, Laurenti R. Impacto do sedentarismo na incidência de doenças crônicas e incapacidades e na ocorrência de óbitos entre os idosos do Município de São Paulo. Saúde Coletiva 2008; 5:183-8.

16. Cunha IC, Peixoto MRG, Jardim PCBV, Alexandre VP. Fatores associados à prática de atividade física na população adulta de Goiânia: monitoramento por meio de entrevistas telefônicas. Rev Bras Epidemiol 2008; 11:495-504.

17. Azevedo MR, Horta BL, Gigante DP, Victora CG, Barros FC. Fatores associados ao sedentarismo no lazer de adultos na coorte de nascimentos de 1982, Pelotas, RS. Rev Saúde Pública 2008; 42 Suppl 2:70-7.

18. Florindo AA, Hallal PC, Moura EC, Malta DC. Prática de atividades físicas e fatores associados em adultos, Brasil, 2006. Rev Saúde Pública 2009; 43 Suppl 2:65-73.

19. Zaitune MPA, Barros MBA, César CLG, Carandina L, Goldbaum M, Alves MCGP. Fatores associados à prática de atividade física global e de lazer em idosos: Inquérito de Saúde no Estado de São Paulo (ISA-SP), Brasil. Cad Saúde Pública 2010; 26 : 1606-18.

20. Rocha SV, Almeida MMG, Araújo TM, Virtuoso JJS. Fatores associados à atividade física no lazer entre residentes de áreas urbanas de um município do nordeste do Brasil. Rev Bras Cineantropom Desempenho Hum 2011; 13:257-64.

21. Suzuki CS, Moraes SA, Freitas ICM. Atividade física e fatores associados em adultos residentes em Ribeirão Preto, SP. Rev Saúde Pública 2011; 45: 311-20.

22. Farias Júnior JC, Lopes AS, Mota J, Hallal PC. Prática de atividade física e fatores associados em adolescentes no Nordeste do Brasil. Rev Saúde Pública 2012; 46:505-15.

23. Porto DB, Guedes DP, Fernandes RA, Reichert FF. Perceived quality of life and physical activity in Brazilian older adults. Motricidade 2012; 8:33-41.

24. Dumith SC. Physical activity in Brazil: a systematic review. Cad Saúde Pública 2009; 25 Suppl 3: S415-26.

25. Alves MCGP, Escuder MML. Plano de amostragem do ISA-Capital 2008. http://www.fsp.usp.br/isasp/pdf/planoamostral2008.pdf (acessado em 28/ Mai/2012).
26. Faculdade de Saúde Pública, Universidade de São Paulo. Inquérito de saúde no Município de São Paulo - ISA-Capital 2008: questionário completo. http://www.fsp.usp.br/isa-sp/pdf/questionari oisa2008.pdf (acessado em 28/Mai/2012).

27. Craig CL, Marshall AL, Sjöström M, Bauman AE, Booth ML, Ainsworth BE, et al. International physical activity questionnaire: 12-country reliability and validity. Med Sci Sports Exerc 2003; 35:1381-95.

28. Nelson ME, Rejeski WJ, Blair SN, Duncan PW, Judge JO, King AC, et al. Physical activity and public health in older adults: recommendation from the American College of Sports Medicine and the American Heart Association. Med Sci Sports Exerc 2007; 39:1435-45.

29. Sofi F, Capalbo A, Marcucci R, Gori AM, Fedi S, Macchi C, et al. Leisure time but not occupational physical activity significantly affects cardiovascular risk factors in an adult population. Eur J Clin Invest 2007; 37:947-53.

30. Hallal PC, Dumith SC, Bastos JP, Reichert FF, Siqueira FV, Azevedo MR. Evolução da pesquisa epidemiológica em atividade física no Brasil: revisão sistemática. Rev Saúde Pública 2007; 41:453-60.

31. Florindo AA, Guimarães VV, Cesar CL, Barros MB, Alves MC, Goldbaum M. Epidemiology of leisure, transportation, occupational, and household physical activity: prevalence and associated factors. J Phys Act Health 2009; 6:625-32.

32. Malta DC, Moura EC, Castro AM, Cruz DKA, Morais NOL, Monteiro CA. Padrão de atividade física em adultos brasileiros: resultados de um inquérito por entrevistas telefônicas, 2006. Epidemiol Serv Saúde 2009; 18:7-16.

33. Azevedo MR, Araujo CL, Reichert FF, Siqueira FV, Silva MC, Hallal PC. Gender differences in leisuretime physical activity. Int J Public Health 2007; 52:8-15.

34. Monteiro CA, Conde WL, Matsudo SM, Matsudo VR, Bonsenor IM, Lotufo PA. A descriptive epidemiology of leisure-time physical activity in Brazil, 1996-1997. Rev Panam Salud Pública 2003; 14: 246-54.

35. Camões M, Lopes C. Fatores associados à atividade física na população portuguesa. Rev Saúde Pública 2008; 42:208-16

36. Browson RC, Jones DA, Pratt M, Blanton C, Heath GW. Measuring physical activity with the behavioral risk factor surveillance system. Med Sci Sports Exerc 2000; 32:1913-8.

37. Pan SY, Cameron C, DesMeules M, Morrison H, Craig CL, Jiang XH. Individual, social, environmental, and physical environmental correlates with physical activity among Canadians: a crosssectional study. BMC Public Health 2009; 9:21.

38. Sousa CA, César CLG, Barros MBA, Carandina L, Goldbaum M, Pereira JCR. Doença pulmonar obstrutiva crônica e fatores associados em São Paulo, SP, 2008-2009. Rev Saúde Pública 2011; 45:887-96. 
39. Varo JJ, Martinez-Gonzalez MA, De Irala-Estévez J, Kearney J, Gibney M, Martínez JA. Distribution and determinants of sedentary lifestyles in the European Union. Int J Epidemiol 2003; 32:138-46.

40. Blair SN. Physical activity, epidemiology, public health and the American College of Sports Medicine. Med Sci Sports Exerc 2003; 35:1463.

41. Chen K, Kandel DB. The natural history of drug use from adolescence to mid-thirties in a general population sample. Am J Public Health 1995; 85:41-7.

42. World Health Organization. The world health report 2002: reducing risks, promoting healthy life. http://www.who.int/whr/2002/en/whr02_en.pdf (acessado em 31/Mai/2012).

43. World Health Organization. Global strategy on diet, physical activity and health. http://www.who. int/dietphysicalactivity/strategy/eb11344/strat egy_english_web.pdf (acessado em 31/Mai/2012).
44. Barreto SM, Pinheiro ARO, Sichieri R, Monteiro CA, Filho MB, Schimidt MI, et al. Análise da estratégia global para alimentação, atividade física e saúde, da Organização Mundial da Saúde. Epidemiol Serv Saúde 2005; 14:41-68.

45. Rzewnicki R, Vanden Auweele Y, De Bourdeaudhuij I. Addressing overreporting on the International Physical Activity Questionnaire (IPAQ) telephone survey with a population sample. Public Health Nutr 2003; 6:299-305.

46. Barros MBA. Inquéritos domiciliares de saúde: potencialidades e desafios. Rev Bras Epidemiol 2008; 11 Suppl 1:6-19.

Recebido em 02/Jul/2012

Versão final reapresentada em 21/Set/2012 Aprovado em 08/Out/2012 